\title{
Latent tuberculosis infection: recent progress and challenges in South Korea
}

\author{
Doosoo Jeon
}

Department of Internal Medicine, Pusan National University Yangsan Hospital, Yangsan, Korea

Received: January 29, 2020 Accepted: February 17, 2020

\section{Correspondence to}

Doosoo Jeon, M.D.

Department of Internal Medicine, Pusan National University Yangsan Hospital, 20 Geumo-ro, Mulgeum-eup, Yangsan 50612 , Korea

Tel: +82-55-360-1414

Fax: +82-55-360-1757

E-mail: sooliıo@hanmail.net https://orcid.org/0000-0002$8206-9487$

This paper was contributed by The Korean Academy of Tuberculosis and Respiratory Diseases.
Management of latent tuberculosis infection (LTBI) is a critical element in the elimination of tuberculosis (TB). However, the low positive predictive value of the current diagnostic tests and the low acceptance and completion rate of the isoniazid-based regimen are major barriers to the implementation and scale-up of programmatic management of LTBI. In the past decade, there has been some progress in the conception, diagnosis, and treatment of LTBI. LTBI is now understood as a dynamic spectrum rather than the traditional binary distinction between active and latent TB. New insight into LTBI has led to a renewed interest in incipient TB, which would be a potential target for developing new diagnostics and therapeutics of LTBI. Recent studies showed that host transcriptomic signatures could be a potential biomarker for incipient TB. The new shorter rifamycin-based regimens have shown comparable efficacy, but better completion rate and safety compared to the isoniazid-based regimen. In South Korea, LTBI management has been expanded and integrated into key elements of the National Tuberculosis Control Program. For the programmatic approach to LTBI management, the following challenges need to be addressed; target group selection, treatment-related interventions, monitoring and surveillance system, and extending the plan for vulnerable groups.

Keywords: Tuberculosis; Latent tuberculosis; Diagnosis

\section{INTRODUCTION}

A world free of tuberculosis (TB) has been humanity's long cherished desire. For decades, TB control programs have focused almost exclusively on infectious TB cases. Active case finding and proper management of active TB should be the top priority for TB control programs. However, it is evident that this strategy alone cannot achieve TB elimination. Despite great efforts, TB is still a major public health threat worldwide, with 10 million new cases and 1.2 million deaths in 2018 [1]. In South Korea, 26,433 new TB cases and 1,816 TB-related deaths were reported in 2018 [2].

Latent tuberculosis infection (LTBI) might be the most important factor for the current uncontrolled TB epidemics. One quarter of the world's population, approximately 1.7 billion people, is estimated to have LTBI [3]. LTBI is a large reservoir for active TB cases because $5 \%$ to $10 \%$ of those infected will develop active TB over their lifetime [4]. How can TB elimination be achieved? Multiple modeling studies have consistently suggested the solution to this critical question. TB elimination can be achieved through comprehensive strategies directed toward integrated LTBI management with proper active TB management $[5,6]$.

Programmatic management of LTBI is emerging as a critical component of TB control programs for TB elimination [7]. According to this paradigm shift, there 
have been many changes and advances in LTBI in the last decade. This paper reviews the recent progress and challenges in the conception, diagnosis, and treatment of LTBI as well as the implementation of LTBI programs in South Korea.

\section{LTBI, AN EVOLVING CONCEPT}

LTBI is defined as a state of persistent immune response to stimulation by Mycobacterium tuberculosis antigens with no evidence of clinically manifest active TB [8]. This definition is based on the traditional concept of a binary distinction between latent and active TB. Recently, LTBI has been understood as a dynamic and continuous spectrum of TB, resulting from the dynamic interaction between the host immune system and TB bacilli [9]. Recent researches suggest new insight on latency, which is dynamic spectrum of TB infection; elimination of $M$. tuberculosis, controlled infection with nonreplicating but viable $M$. tuberculosis, replicating $M$. tuberculosis at subclinical level, and actively replicating M. tuberculosis $[9,10]$. New insight on LTBI has led to renewed interest in the 'incipient' phase of TB [11]. Incipient TB is defined as an infection with viable $M$. tuberculosis bacteria that is likely to progress to active TB but has not yet induced clinical symptoms, radiographic abnormalities, or microbiologic evidence consistent with active TB disease [12]. Therefore, patients with incipient TB who are very likely to progress to active TB shortly would be an attractive target group with the greatest benefit for LTBI treatment [13]. A better understanding of incipient TB would accelerate the development of more predictive diagnostic tests and effective therapeutic interventions for LTBI.

\section{DIAGNOSIS, NEW TESTS}

The tuberculin skin test (TST) and interferon gamma release assays (IGRAs) are the main diagnostic tests for LTBI. Both are indirect tests based on immune response to TB and do not directly assess the presence or viability of TB bacilli. Both tests cannot differentiate between various stages of TB infection, and thus have a poor positive predictive value (PPV) to predict progres- sion to active TB $[14,15]$. This leads to a high number of individuals who would need to be treated in order to prevent one case of active TB [16]. In a meta-analysis, the pooled PPV of the TST to predict active TB within 2 years was $1.5 \%$ and the number needed to treat (NNT) in order to prevent one TB case was 67.39; IGRAs performed slightly better, with a PPV of $2.7 \%$ and an NNT of 37.3 [17]. Due to the limitations of currently available tests, many individuals could not avoid unnecessary treatment and thus, face the potential risk of adverse events such as hepatotoxicity. New diagnostic tests with high predictive value to predict progression to active TB are urgently needed.

\section{QuantiFERON-TB Plus}

QuantiFERON-TB Plus (QFT-Plus) is the new version of the QFT-Gold In-Tube. Compared to the QFT-Gold In-Tube containing antigens optimized to stimulate $\mathrm{CD}_{4}{ }^{+} \mathrm{T}$ cells, QFT-Plus contains new antigens optimized for both $\mathrm{CD}_{4}{ }^{+}$and $\mathrm{CD} 8^{+} \mathrm{T}$ cell stimulation [18]. QFT-Plus has potential to indicate recent infection and disease activity. A recent meta-analysis has revealed that QFT-Plus is a more sensitive test compared to QFTGIT for detecting M. tuberculosis infection [19].

\section{C-Tb test}

The $\mathrm{C}$ - $\mathrm{Tb}$ test is a recently developed skin test utilizing early secreted antigenic target 6 (ESAT-6) and culture filtrate protein 10 (CFP-10) antigens. The C-Tb test is as simple as conducting a TST but has a higher specificity. In several phase III trials of C-Tb [20,21], C-Tb positivity using a 5 -mm cutoff correlated with exposure risk, performed better than TST in Bacillus CalmetteGuérin (BCG)-vaccinated people, and had a high correlation with the results of the QFT-GIT.

\section{Biomarkers for incipient TB}

The key purpose of diagnosing LTBI is to identify who is very likely to progress to active TB [22]. Incipient TB is an attractive target for the development of new diagnostics. Host blood transcriptomic mRNA signatures have emerged as a promising platform for discovering biomarkers for incipient TB [23]. Recent studies have shown that transcriptomic signatures can predict the development of active TB $[24,25]$. These studies analyzed whole blood RNA sequencing data from large 
cohorts and revealed that some combinations of gene signatures showed a good correlation with the risk for progression to active TB in 6 to 12 months. Further research is needed for its validation and further development as a point-of-care test.

\section{PROGRESS IN TREATMENTS}

\section{New shorter regimens}

Isoniazid has been the main drug for LTBI treatment during the past decades. However, the 6 to 9 months of isoniazid regimen has shown low acceptance and completion rates due to the long treatment duration and poor tolerability $[26,27]$. These are the main barriers in the implementation and scale up of the programmatic LTBI treatment.

Recent studies have shown that shorter regimens using rifampicin or rifapentine are as effective as isoniazid-based regimens with higher completion rates and better safety [28-30]. A daily dose of rifampicin for 4 months [28] and 12 weeks of weekly isoniazid and rifapentine [29] were not inferior to 9 months of isoniazid for the prevention of active TB. Both regimens also showed better completion rates and fewer serious adverse events, especially hepatitis. Another much shorter regimen, one month of daily isoniazid and rifapentine [30], was not inferior to 9 months of isoniazid with a higher completion rate. Shorter rifamycin-based regimens may replace 6-9 months of isoniazid, considering better safety and adherence [31].

However, a recent Korean study of a treatment regimen using 12-week of weekly isoniazid and rifapentine showed a higher rate of flu-like symptoms and a 1.8\% rate of anaphylaxis, despite a higher completion rate and lesser hepatotoxicity than 3 months of isoniazid and rifampicin [32]. These findings raise concerns that this regimen might not be appropriate for LTBI regimen for the general population in South Korea.

\section{New vaccine}

Therapeutic vaccination is a new and promising approach for LTBI treatment. In a phase IIb trial, a candidate vaccine, $\mathrm{M}_{72} / \mathrm{ASO}_{\mathrm{E}}$, reduced the risk of developing active TB in human immunodeficiency virus (HIV)-negative people with LTBI by $49 \cdot 7 \%$ for 3 years [33].
The overall efficacy of $49.7 \%$ is low compared with that of most established vaccines in the treatment of other diseases. However, considering the high prevalence of LTBI worldwide and the limitations of current preventive chemotherapy, a vaccine could have a great impact on TB control. In addition, the vaccine can be expected to work in patients with drug-resistant TB who show the limited effect of preventive chemotherapy.

\section{LTBI IN SOUTH KOREA}

\section{Changes in LTBI prevalence}

The prevalence of LTBI in Korea has gradually decreased in accordance with a decrease in the prevalence of TB. According to the National Tuberculosis Prevalence Survey, which was conducted every 5 years from 1965 to 1995 , the LTBI prevalence in general population decreased from $64.2 \%$ in 1965 to $59.3 \%$ in 1975 then to $44.4 \%$ in 1990 (Fig. 1) [34]. In 2016, the Korea National Health and Nutrition Examination Survey showed an LTBI prevalence of $33.2 \%$ [35].

One of the key changes in the LTBI prevalence is its remarkable decrease in the younger population. The LTBI prevalence among those aged $<30$ years was $44.5 \%$ in 1965 , which was decreased to $15.5 \%$ in 1995 [36]. Recent studies showed further decrease in the rate of LTBI in the younger population (Table 1). In the 2016 surveillance using TST, the LTBI prevalence was $6.5 \%$ and $10.9 \%$ in the ages 10 to 19 and 20 to 29 , respectively [35], and which was further decreased $2.0 \%$ and $3.4 \%$, respectively, in the 2018 surveillance using IGRA [37].

In contrast, the prevalence of LTBI in the elderly population is still high. Many studies in various settings have shown that age is the most important predictor of LTBI [37-39]. To implement and scale up the programmatic management of LTBI in South Korea, the age differences of the LTBI prevalence should be considered.

\section{Progress in the LTBI program}

In South Korea, the National Tuberculosis Control policies have focused on early diagnosis and appropriate treatment of patients with active TB [34]. Recently LTBI management has been expanded and integrated into key elements of the National Tuberculosis Con- 
Table 1. The rate of latent tuberculosis infection by age group in 2016 to 2018

\begin{tabular}{lccc}
\hline \multirow{2}{*}{ Age group, yr } & \multicolumn{3}{c}{ Year } \\
\cline { 2 - 4 } & $2016[35]^{\mathrm{a}}$ & $2017[38]^{\mathrm{b}}$ & $2018[37]^{\mathrm{c}}$ \\
\hline $10-19$ & 6.5 & $\mathrm{NA}$ & 3.0 \\
$20-29$ & 10.9 & 5.5 & 3.4 \\
$30-39$ & 36.4 & 12.7 & 9.1 \\
$40-49$ & 46.1 & 23.0 & 17.7 \\
$50-59$ & 48.7 & 35.1 & 25.4 \\
$\geq 60$ & 45.0 & 42.0 & 31.0 \\
Total & 33.2 & 20.3 & 13.2 \\
\hline
\end{tabular}

Values are presented as percentage.

NA, not applicable.

${ }^{\mathrm{a}}$ Results from general population $(\mathrm{n}=2,051)$, by tuberculin skin test.

${ }^{\mathrm{b}}$ Results from facility workers in congregated settings ( $\mathrm{n}=$ $543,538)$, by interferon-gamma releasing assay.

${ }^{\mathrm{c}}$ Results from facility workers in congregated settings $(\mathrm{n}=$ $128,906)$, by interferon-gamma releasing assay.

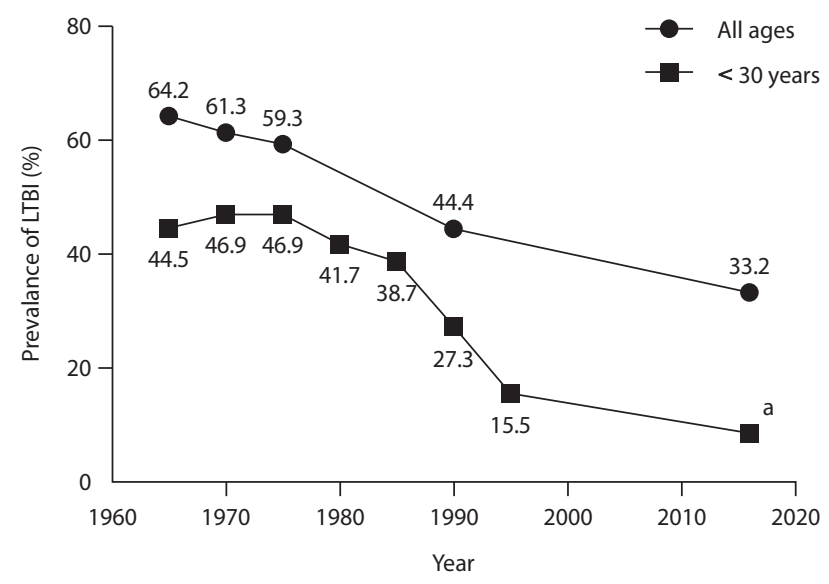

Figure 1. Trend in the prevalence of latent tuberculosis infection in South Korea. This figure was drawn based on the references [34-36]. LTBI, latent tuberculosis infection. ${ }^{\mathrm{a}} 6.5 \%$ and $10.9 \%$ in the ages $10-19$ and $20-29$, respectively.

trol program [34,40]. An LTBI program was started for HIV-infected persons and household contacts under 6 years of age in 2004 [40]. Contact investigation programs were expanded to close contacts under 18 years of age in 2008, and to all age groups in 2011. In 2013, a TB epidemiologic investigation team was established, and active contact investigation was expanded to close contact in congregate settings. In 2016, the Tuberculosis Prevention Act was amended, making TB and latent
TB screening mandatory for community workers in congregate settings. By the Tuberculosis Prevention Act, systematic LTBI screening and treatment were conducted for about 1.2 million individuals in congregated settings in 2017 [38].

LTBI management has been recommended as an independent chapter in the 2011 Korean TB guidelines [41]. In the updated 2017 Korean TB guidelines, the age limit for LTBI treatment among close contacts was extended from 35 to 65 years [41]. In addition, screening for and treatment of LTBI in healthcare workers was first recommended.

\section{Challenges}

In the past decade, the LTBI program has been expanded and progressed under the vision of 'TB Free Society, Healthy Country.' It is time to take a step forward to implement and scale-up programmatic LTBI management. For a programmatic approach, the following challenges need to be addressed; target group selection, treatment-related interventions, monitoring and surveillance system, and an extension plan for vulnerable and hard-to-reach groups.

Prioritization of the target population should be the first step in a national plan for programmatic management of LTBI $[8,42]$. The most important benefit of LTBI treatment is 'individual-level benefit,' which reduces the risk of developing TB in high-risk individuals. Another key benefit is 'public health-level benefit' which could reduce further transmission of TB in the community [43]. This is an important reason for offering LTBI screening and treatment to workers in congregated settings, including healthcare workers. To achieve TB elimination, a public health approach in LTBI management will be necessary. However, this approach has concerns about increasing the individual risk of harm from unnecessary treatment. In addition, the impact of this approach on overall TB control should be carefully assessed to optimize the allocation of available resources. In 2017, there was a debate about the plan of LTBI mass screening for adults aged 40 and first-grade high school students [44]. The Korean government conducted the proactive LTBI screening and treatment among workers in congregate settings for about 1.2 million individuals in 2017 and 0.5 million individuals in 2018 [37,38]. This project includes several 


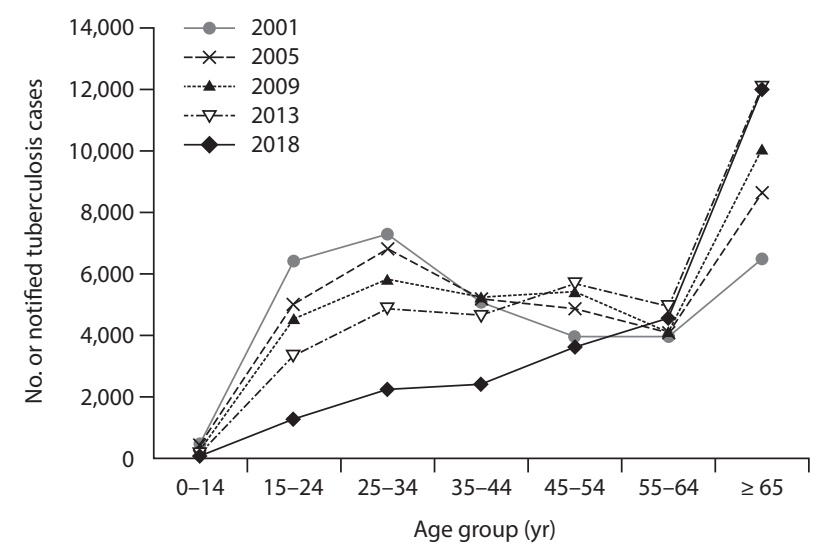

Figure 2. Trends in age-specific cases of new tuberculosis in South Korea from the years 2001 to 2018 . Adopted from Lee [49], according to Creative Commons License.

groups not recommended for high-risk groups by the WHO [8]. The achievement and limitation of this project should be carefully reviewed to develop strategies that are optimized for the Korean situation.

Proper management of those identified as LTBI cases is also important. Low acceptance and poor completion rate are the major barriers to implementing programmatic LTBI management [26,27]. In order to increase adherence to LTBI treatment, treatment-related interventions should be accompanied, such as case management, education, and counselling. Appropriate recording, reporting, and monitoring systems should be established.

In addition, plans for vulnerable and hard-to-reach groups (i.e., elderly individuals, foreigners residing in Korea [45], North Korean defectors [46], and the homeless) should be explored $[47,48]$. TB among the elderly population is becoming a major problem in TB control, which accounts for approximately $40 \%$ of new TB cases and $78 \%$ of TB-related deaths in South Korea. The number of new TB cases in $\geq 65$ years of age has increased consistently with time (Fig. 2). In this group, reactivation from past infection is the major mechanism of developing active TB rather than recent transmission. Despite its theoretical benefit, providing LTBI treatment to individuals $\geq 65$ years is a clinical challenge because of the high risk of age-related adverse events such as hepatotoxicity. Therefore, a tailored approach to LTBI treatment in the elderly population is needed. Active case finding through sys- temic screening is another approach to managing TB in the elderly [49]. Recently, a pilot project of systematic TB screening in the elderly showed a high rate of TB detection [50].

\section{CONCLUSIONS}

LTBI treatment is a paradigm shift in TB control strategies aimed at the elimination of TB. Much progress has been made in the last decade. New insight into LTBI has led to the development of new biomarkers that have the potential to identify the target group that is very likely to progress to active TB. The shorter rifamycin-based regimens have been proven to be effective, safe, and tolerable, which could enhance adherence and completion and minimize adverse events. Along with this progress, to eliminate TB, further effort is needed to implement and expand the programmatic management of LTBI.

\section{Conflict of interest}

No potential conflict of interest relevant to this article was reported.

\section{REFERENCES}

1. World Health Organization. Global tuberculosis report 2019 [Internet]. Geneva (CH): World Health Organization, 2018 [cited 2020 Jan 25]. Available from: https://www.who. int/tb/publications/global_report/en/.

2. Korea Centers for Disease Control and Prevention. Annual report on the notified tuberculosis patients in Korea 2018 [Internet]. Cheongju (KR): Korea Centers for Disease Control and Prevention, 2019 [cited 2020 Jan 25]. Available from: http://tbzero.cdc.go.kr/tbzero/board/boardView.do.

3. Houben RM, Dodd PJ. The global burden of latent tuberculosis infection: a re-estimation using mathematical modelling. PLoS Med 2016;13:e1002152.

4. Comstock GW, Livesay VT, Woolpert SF. The prognosis of a positive tuberculin reaction in childhood and adolescence. Am J Epidemiol 1974;99:131-138.

5. Dye C, Glaziou P, Floyd K, Raviglione M. Prospects for tuberculosis elimination. Annu Rev Public Health 2013;34:271-286. 
6. Menzies NA, Cohen T, Hill AN, et al. Prospects for tuberculosis elimination in the United States: results of a transmission dynamic model: version 2. Am J Epidemiol 2018;187:2011-2020.

7. Uplekar M, Weil D, Lonnroth K, et al. WHO's new end TB strategy. Lancet 2015;385:1799-1801.

8. World Health Organization. Latent TB infection: updated and consolidated guidelines for programmatic management [Internet]. Geneva (CH): World Health Organization, 2018 [cited 2020 Jan 25] Available from: https://www. who.int/tb/publications/2018/latent-tuberculosis-infection/en/.

9. Barry CE 3rd, Boshoff HI, Dartois V, et al. The spectrum of latent tuberculosis: rethinking the biology and intervention strategies. Nat Rev Microbiol 2009;7:845-855.

10. Esmail H, Barry CE 3rd, Wilkinson RJ. Understanding latent tuberculosis: the key to improved diagnostic and novel treatment strategies. Drug Discov Today 2012;17:514521.

11. Achkar JM, Jenny-Avital ER. Incipient and subclinical tuberculosis: defining early disease states in the context of host immune response. J Infect Dis 2011;204 Suppl 4:S1179-S1186.

12. Drain PK, Bajema KL, Dowdy D, et al. Incipient and subclinical tuberculosis: a clinical review of early stages and progression of infection. Clin Microbiol Rev 2018;31:eooo21-18.

13. World Health Organization. Consensus meeting report: development of a target product profile (TPP) and a framework for evaluation for a test for predicting progression from tuberculosis infection to active disease [Internet]. Geneva (CH): World Health Organization, 2017 [cited 2020 Jan 25]. Available from: https://www.who.int/tb/ publications/2017/tpp_infection_disease/en/.

14. Haas MK, Belknap RW. Diagnostic tests for latent tuberculosis infection. Clin Chest Med 2019;40:829-837.

15. Overton K, Varma R, Post JJ. Comparison of interferon- $\gamma$ release assays and the tuberculin skin test for diagnosis of tuberculosis in human immunodeficiency virus: a systematic review. Tuberc Respir Dis (Seoul) 2018;81:59-72.

16. Matteelli A, Lonnroth K, Getahun H, Falzon D, Migliori GB, Raviglione M. Numbers needed to treat to prevent tuberculosis. Eur Respir J 2015;46:1838-1839.

17. Diel R, Loddenkemper R, Nienhaus A. Predictive value of interferon- $\gamma$ release assays and tuberculin skin testing for progression from latent TB infection to disease state: a meta-analysis. Chest 2012;142:63-75.

18. Kim SH, Jo KW, Shim TS. QuantiFERON-TB Gold PLUS versus QuantiFERON- TB Gold In-Tube test for diagnosing tuberculosis infection. Korean J Intern Med 2020;35:383-391.

19. Sotgiu G, Saderi L, Petruccioli E, et al. QuantiFERON TB Gold Plus for the diagnosis of tuberculosis: a systematic review and meta-analysis. J Infect 2019;79:444-453.

20. Aggerbeck H, Ruhwald M, Hoff ST, et al. C-Tb skin test to diagnose Mycobacterium tuberculosis infection in children and HIV-infected adults: a phase 3 trial. PLoS One 2018;13:e0204554.

21. Ruhwald M, Aggerbeck H, Gallardo RV, et al. Safety and efficacy of the C-Tb skin test to diagnose Mycobacterium tuberculosis infection, compared with an interferon $\gamma$ release assay and the tuberculin skin test: a phase 3, double-blind, randomised, controlled trial. Lancet Respir Med 2017;5:259-268.

22. Goletti D, Lee MR, Wang JY, Walter N, OttenhoffTH. Update on tuberculosis biomarkers: from correlates of risk, to correlates of active disease and of cure from disease. Respirology 2018;23:455-466.

23. Singhania A, Wilkinson RJ, Rodrigue M, Haldar P, O'Garra A. The value of transcriptomics in advancing knowledge of the immune response and diagnosis in tuberculosis. Nat Immunol 2018;19:1159-1168.

24. Suliman S, Thompson E, Sutherland J, et al. Four-gene pan-African blood signature predicts progression to tuberculosis. Am J Respir Crit Care Med 2018;197:1198-1208.

25. Penn-Nicholson A, Hraha T, Thompson EG, et al. Discovery and validation of a prognostic proteomic signature for tuberculosis progression: a prospective cohort study. PLoS Med 2019;16:e1002781.

26. Alsdurf H, Hill PC, Matteelli A, Getahun H, Menzies D. The cascade of care in diagnosis and treatment of latent tuberculosis infection: a systematic review and meta-analysis. Lancet Infect Dis 2016;16:1269-1278.

27. Kim HW, Kim JS. Treatment of latent tuberculosis infection and its clinical efficacy. Tuberc Respir Dis (Seoul) 2018;81:6-12.

28. Menzies D, Adjobimey M, Ruslami R, et al. Four months of rifampin or nine months of isoniazid for latent tuberculosis in adults. N Engl J Med 2018;379:440-453.

29. Sterling TR, Scott NA, Miro JM, et al. Three months of weekly rifapentine and isoniazid for treatment of Mycobacterium tuberculosis infection in HIV-coinfected per- 
sons. AIDS 2016;30:1607-1615.

30. Swindells S, Ramchandani R, Gupta A, et al. One month of rifapentine plus isoniazid to prevent HIV-related tuberculosis. N Engl J Med 2019;380:1001-1011.

31. Campbell JR, Trajman A, Cook VJ, et al. Adverse events in adults with latent tuberculosis infection receiving daily rifampicin or isoniazid: post-hoc safety analysis of two randomised controlled trials. Lancet Infect Dis 2019 Dec 19 [Epub]. https://doi.org/10.1016/S1473-3099(19)30575-4.

32. Jo KW, Kim JS, Kwon HS, et al. Adverse event and treatment completion rates of a 12-dose weekly isoniazid and rifapentine course for South Korean healthcare workers. Respir Med 2019;158:42-48.

33. Tait DR, Hatherill M, van Der Meeren O, et al. Final analysis of a trial of M72/ASo1E vaccine to prevent tuberculosis. N Engl J Med 2019;381:2429-2439.

34. Cho KS. Tuberculosis control in the Republic of Korea. Epidemiol Health 2018;40:e2018036.

35. Korean National Tuberculosis Association. 7th Korea National Health and Nutrition Examination Survey, 1st Year (2016): Tuberculin Survey Support and Quality Control. Seoul (KR): Korea Centers for Disease Control and Prevention, 2017.

36. Kim SJ, Hong YP, Lew WJ, Yang SC, Lee EG. Incidence of pulmonary tuberculosis in Korean civil servants. Tuber Lung Dis 1995;76:534-539.

37. Choi JK, Park AY, Jang MR, et al. The prevalence of latent tuberculosis infection in congregated settings in the Republic of Korea, 2018. Public Health Wkly Rep 2019;12:786-792.

38. Cho KS, Park WS, Jeong HR, Kim MJ, Park SJ, Park AY. Prevalence of latent tuberculosis infection at congregated settings in the Republic of Korea, 2017. Public Health Wkly Rep 2018;11:348-354.

39. Park JS. The prevalence and risk factors of latent tuberculosis infection among health care workers working in a tertiary hospital in South Korea. Tuberc Respir Dis (Seoul)
2018;81:274-280.

40. Go U, Park M, Kim UN, et al. Tuberculosis prevention and care in Korea: evolution of policy and practice. J Clin Tuberc Other Mycobact Dis 2018;11:28-36.

41. Lee SH. Diagnosis and treatment of latent tuberculosis infection: the updated 2017 Korean guidelines. Korean J Med 2018;93:509-517.

42. Rangaka MX, Cavalcante SC, Marais BJ, et al. Controlling the seedbeds of tuberculosis: diagnosis and treatment of tuberculosis infection. Lancet 2015;386:2344-2353.

43. Campbell JR, Dowdy D, Schwartzman K. Treatment of latent infection to achieve tuberculosis elimination in low-incidence countries. PLoS Med 2019;16:e1002824.

44. Bae JM. An opposing view on including high school students in a latent tuberculosis infection control program in Korea. Epidemiol Health 2017;39:e2017015.

45. Lee S, Ryu JY, Kim DH. Pre-immigration screening for tuberculosis in South Korea: a comparison of smearand culture-based protocols. Tuberc Respir Dis (Seoul) 2019;82:151-157.

46. Kim BK, Kim HJ, Kim HJ, et al. Experiences of latent tuberculosis infection treatment for the North Korean refugees. Tuberc Respir Dis (Seoul) 2019;82:306-310.

47. Kim JK, Jeong I, Lee JY, et al. Tuberculosis relief belt supporting project (tuberculosis patient management project for poverty group). Tuberc Respir Dis (Seoul) 2018;81:241246.

48. Kim HW, Kim JS. One step toward a low tuberculosis-burden country: screening for tuberculosis infection among the immigrants and refugees. Tuberc Respir Dis (Seoul) 2020;83:104-105.

49. Lee $\mathrm{SH}$. Active case finding in the elderly tuberculosis in South Korea. Tuberc Respir Dis (Seoul) 2019;82:261-263.

50. Kim H, Kim HJ, Oh KH, Oh HW, Choi H. A pilot project of systematic tuberculosis screening in the elderly in a South Korean province. Tuberc Respir Dis (Seoul) 2019;82:194-200. 